\title{
CONTINUOUS FIREFLY ALGORITHM FOR OPTIMAL TUNING OF PID CONTROLLER IN AVR SYSTEM
}

\author{
Omar Bendjeghaba
}

\begin{abstract}
This paper presents a tuning approach based on Continuous firefly algorithm (CFA) to obtain the proportional-integralderivative (PID) controller parameters in Automatic Voltage Regulator system (AVR). In the tuning processes the CFA is iterated to reach the optimal or the near optimal of PID controller parameters when the main goal is to improve the AVR step response characteristics. Conducted simulations show the effectiveness and the efficiency of the proposed approach. Furthermore the proposed approach can improve the dynamic of the AVR system. Compared with particle swarm optimization (PSO), the new CFA tuning method has better control system performance in terms of time domain specifications and set-point tracking.

Key w ords: automatic voltage regulator(AVR), continuous firefly algorithm (CFA), PID controller, power system security
\end{abstract}

\section{INTRODUCTION}

In modern secure power system, damping of generator terminal voltage oscillation following disturbances is of great concern because it can seriously affect the security of the entire power system. In practice, this role is devoted to the generator excitation system in order to maintain generator voltage and to control the reactive power flow using an automatic voltage regulator (AVR) [1]. Hence, with the advancement in the design of fast acting AVRs as well as the increasing complexity of large interconnected power systems, oscillations may continue for an extended period and even instability may occur following some system disturbances.

Despite the potential of the modern control techniques with different structure, Proportional Integral Derivative (PID) type controller is still widely used for AVR system [2]. Industrial implementations of PID controllers in AVR systems show that the appropriate selection of PID controller parameters results in satisfactory performance during system upsets. Thus, the optimal tuning of a PID gains is required to get the desired level of robust performance. Since optimal setting of PID controller gains is a multimodal optimization problem and more complex due to nonlinearity and time-variability of real world power system operation. Therefore, the traditional techniques are not completely systemic and most of them occasionally yield poor performance in practice, so they are not suitable for such a problem.

Recently, metaheuristic approaches, have received increased attention from researchers dealing with AVR's control problems. In 2004, a PSO based PID type controller for AVR system was presented By Giang [3] to find the optimal parameters of the PID controller so that the desired system specifications are satisfied. Kim and Cho developed an optimal tuning method using hybrid Genetic Algorithm (GA) and Bacterial Foraging (BF) tech- nique to improve the performance of PID control of AVR system [4]. In order to obtain an optimal PID controller for an AVR, Mukherjee and Ghoshal presented a craziness based particle swarm optimization (CRPSO) and binary coded genetic algorithm (GA) [5]. Ching-Chang suggested a real-valued genetic algorithm (RGA) and a particle swarm optimization (PSO) to design PID controller for AVR system [6].

More recently, Shayeghi and Dadashpour presented an anarchic society optimization based PID control of an Automatic voltage regulator system [7]. In this paper, an efficient tuning approach is proposed to find the optimal PID parameters, and a practical high order AVR system with a PID controller is adopted to investigate the performance of the proposed CFA-PID controller.

The approach is based on one of the recent metaheuristic optimization methods inspired by the real fireflies' behaviour, namely the firefly algorithm. The idea of employing the cooperating fireflies agents to solve optimization problems was introduced by Xin-She Yang at Cambridge University and presented in [8] then extended in his further work [9]. It has been, recently, adapted for solving various design problems [10-12]. The FA has been successfully applied to the Permutation Flow Shop Scheduling Problems in [10], to the Clustering problem in [11], and to Mixed variable structural optimization in [12]. Firefly algorithm achives very good results in many applied areas. The FA has also been adapted with success to other combinatorial optimization problems $[13,14]$.

\section{LINEARIZED MODEL OF AN AVR SYSTEM WITH PID CONTROLLER}

\subsection{Pid controller}

The PID controller is used to improve the dynamic response as well as to reduce or eliminate the steady-

* LREEI ,Department of Automatic Control of Industrial Processes and Electrification,Faculty of Sciences, University MHamed Bougara of Boumerdes, Independency Avenue, 35000 Algeria, bendjeghaba@umbb.dz 


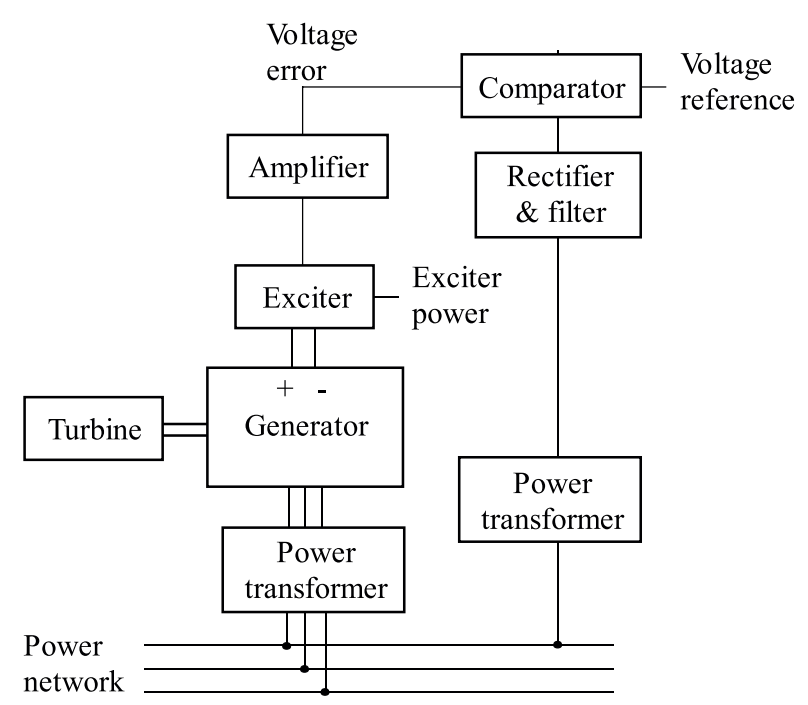

Fig. 1. Real model of an AVR system [1]

state error. The derivative controller adds a finite zero to the open-loop plant transfer function and improves the transient response. The integral controller adds a pole at the origin and increases the system order by one and reduces the steady-state error due to a step function to zero. The PID controller transfer function is done by

$$
G_{P I D}(s)=k_{P}+\frac{k_{I}}{s}+K_{D} s
$$

\subsection{Linearized model of an AVR}

The AVR system contains four main components which are: amplifier, exciter, generator, sensor. Thus, the real model of such system is depicted in Fig. 1 [1].

In order to model the four aforementioned components and determine their transfer functions. Each component must be linearized taking into account the major time constant and ignores the saturation or other nonlinearities. The approximate transfer functions of these components may be represented, respectively, as follows [3].

\section{- Amplifier model}

The amplifier is represented by a gain $K_{a}$ and a time constant $\tau_{a}$, and the transfer function is

$$
\frac{V_{R}(S)}{V_{e}(S)} \frac{K_{a}}{1+\tau_{a} S} .
$$

\section{- Exciter model}

In the simplest form, the transfer function of a modern exciter may be represented by a single time constant $\tau_{e}$ and a gain $K_{e}$ :

$$
\frac{V_{F(S)}}{V_{R}(S)}=\frac{K_{e}}{1+\tau_{e} S}
$$

The time constant of modern exciters are very small.

\section{- Generator model}

In the linearized model, the transfer function relating the generator terminal voltage to its field voltage can be represented by a gain $K_{g}$ and a time constant $\tau_{g}$ and the transfer function is

$$
\frac{V_{t}(S)}{V_{F}(S)}=\frac{K_{g}}{1+\tau_{g} S} .
$$

These constants are load-dependent

- Sensor model

The sensor is modeled by a simple first order transfer function, given by

$$
\frac{V_{s}(S)}{V_{t}(S)}=\frac{K_{s}}{1+\tau_{s} S} .
$$

Utilizing the above models, the AVR block diagram compensated with PID is shown in Fig 2.

\section{BASICS OF FIREFLY ALGORITHM}

Continuous Firefly Algorithm (CFA) is a nature inspired algorithm, which is based on the flashing light of fireflies. In fact, the algorithm has three particular idealized rules which are based in real on some major flashing characteristics of real fireflies [8]. These are the following: (1) all fireflies are unisex, and they will move towards more attractive and brighter ones regardless their sex. (2) the degree of attractiveness of a firefly is proportional to its brightness which decreases as the distances from the other fireflies increases. (3) If there is no brighter or more attractive firefly then a particular one, then it will move randomly. For an optimization problem, the flashing light is associated with the fitness function in order to obtain efficient optimal solutions. The main steps of standard

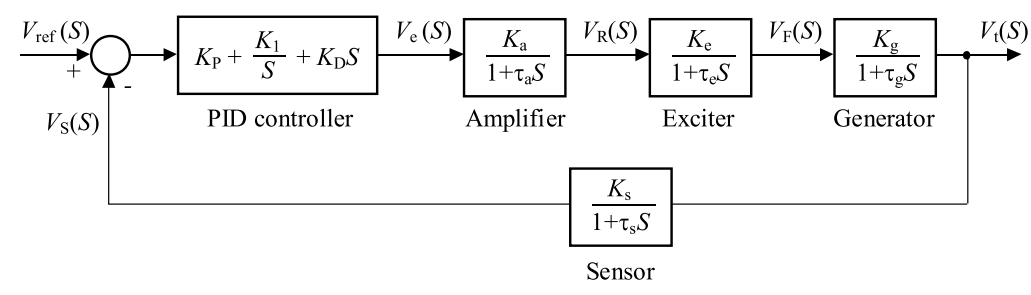

Fig. 2. Block diagram of an AVR system 


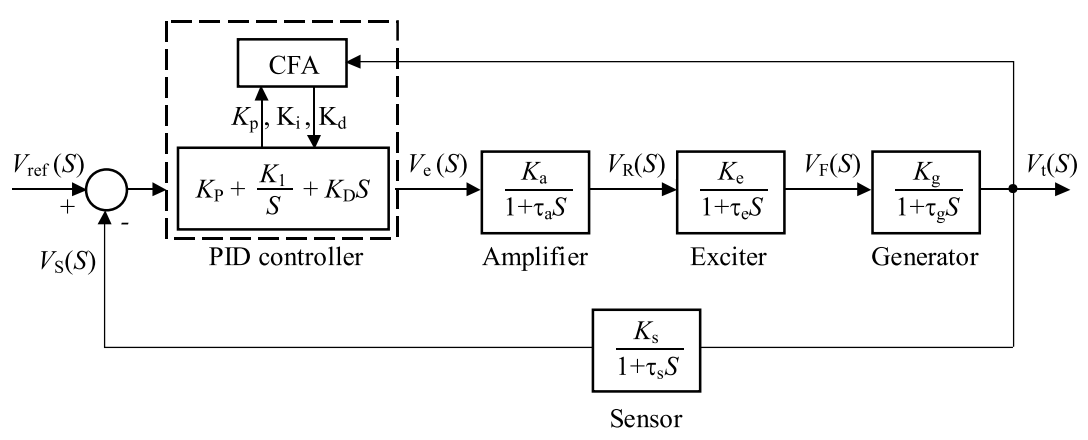

Fig. 3. Block diagram of an AVR system with CFA-PID controller

Table 1. Pseudo code of the CFA Metaheuristic

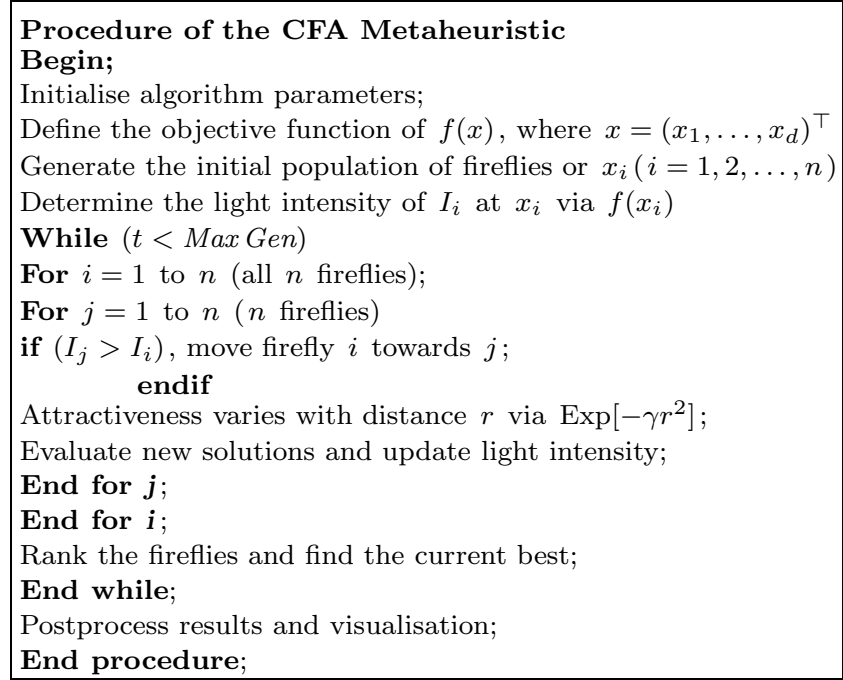

continuous firefly algorithm are given by the pseudo code shown in Tab. 1

When searching for solutions the fireflies use two main procedures: attractiveness and movement, which are defined as follows $[8,9]$.

\section{- Attractiveness}

The form of the attractiveness function of a firefly is the following monotonically decreasing function

$$
\beta(r)=\beta_{0} \exp \left(-\gamma r^{m}\right) \text { with } m \geqslant 1
$$

where $r$ is the distance between any two fireflies, $\beta_{0}$ is the initial attractiveness at $r=0$, and $\gamma$ is the absorption parameter which controls the decrease of the light intensity.

The distance $r$ between any two fireflies $i$ and $j$ at position $x_{i}$ and $x_{j}$, respectively, can be defined as a Cartesian or Euclidean as follows

$$
r_{i j}=\sqrt{\sum_{k=1}^{d}\left(x_{i, k}-x_{j, k}\right)^{2}}
$$

where $x_{i, k}$ is the $k^{\text {th }}$ component of the spatial coordinate $x_{i}$ of the $i^{\text {th }}$ firefly and $d$ is the dimensions number.
- Movement

The movement of a firefly $i$ which is attracted by a brighter firefly $j$ is given by

$$
x_{i}=x_{i}+\beta_{0} e^{-\gamma r_{i j}^{2}}\left(x_{j}-x_{i}\right)+\alpha\left(\operatorname{rand}-\frac{1}{2}\right)
$$

where the first term is the current position of a firefly, the second term is used for considering a firefly's attractiveness to light intensity seen by adjacent fireflies, and the third term is used for the random movement of a firefly in case there are not any brighter ones. The coefficient $\alpha$ is a randomization parameter determined by the problem of interest, while rand is a random number generator uniformly distributed in the space $[0,1]$.

\section{IMPLEMENTATION OF CFA-PID CONTROLLER}

In this paper, a PID controller using CFA was proposed to improve the dynamic of an AVR system. The block diagram of a practical AVR system using CFA-PID is shown in Fig. 3.

\subsection{Fitness function definition}

To improve the step transient response of an AVR system, the main goal of the proposed CFA-PID controller is to adjust optimally as fast as possible the PID controller parameters by minimization of predetermined fitness function. In time domain, the fitness (objective function) can be formed by different performance specifications such as the integral of time multiplied by absoluteerror value (ITAE), rise time, settling time, overshoot and steady state error. In this paper and for the purpose of comparison, the following performance function is used [6]

$$
F(K)=I T A E\left(\left(1-\mathrm{e}^{\rho}\right)\left(M_{p}+E_{s s}\right)+\mathrm{e}^{\rho}\left(T_{s}-T_{r}\right)\right)
$$

where $K=\left[K_{P}, K_{I}, K_{D}\right]$ is a parameter set of PID controller, $\rho$ is a weighting factor, ITAE, $M_{p}, E_{s s}, T_{s}$ and $t_{r}$ are respectively the integral of time multiplied by absolute-error value, the maximum overshoot, the steady state error, the settling time and the rising time of the performance criteria in the time domain. 


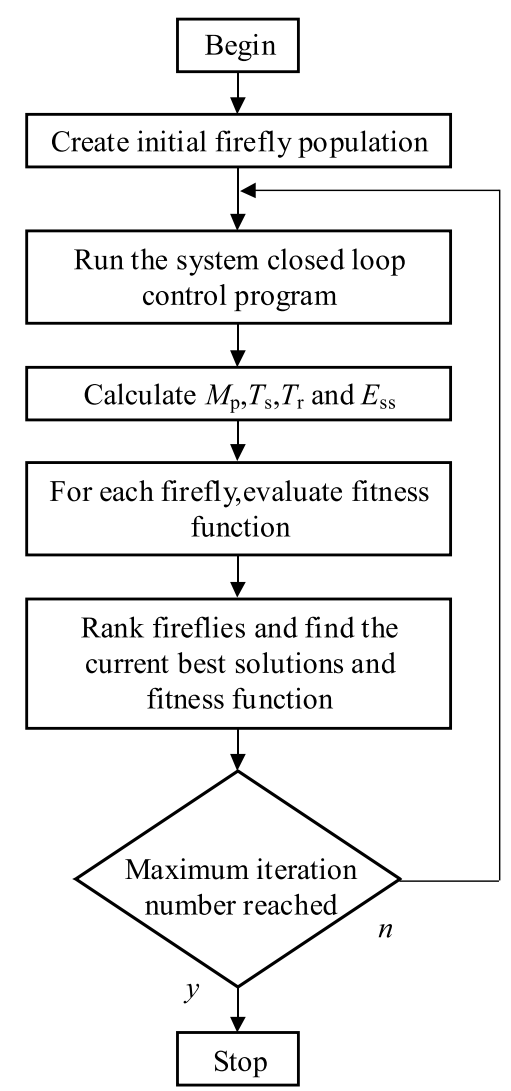

Fig. 4. Flow chart of the proposed CFA-PID controller

Table 2. CFA parameters values

\begin{tabular}{ccc}
\hline Parameter & designation & value \\
\hline$n$ & number of fireflies & 20 \\
$\alpha$ & randomness & .15 \\
$\beta_{0}$ & Initial Attractiveness & .1 \\
$\gamma$ & Absorption coefficient & 1 \\
$\delta$ & Weighting factor & 0.9 \\
$n g$ & generation number & 100 \\
\hline
\end{tabular}

\subsection{CFA-PID mechanism in AVR system}

Similarly to other metaheuristics optimization methods, firefly algorithm generates initial random population of feasible candidate solutions. All fireflies of the population are handled in the solution search space with the aim to guide the search to the best location in the search space using their flashing light (Fitness) and randomness. Each firefly in the population moves in the three-dimensional (PID parametres) search space with an attractiveness which is dynamically updated based on the knowledge of the firefly and its neighbours. In this paper and conversely to the standard CFA, We note that the randomness factor is iteratively reduced in order to accelerate the algorithm convergence according to this equation

$$
\alpha_{t+1}=\alpha_{t} \delta e^{\sqrt{t}}
$$

where $t$ is the iteration counter, $\delta$ is a positive weighting factor.
Applying this search mechanism over the iterations, the CFA find looks for the optimal set of PID parameters, while minimizing the fitness function. The flow chart of the proposed CFA for PID tuning is shown in Fig. 4.

\section{SIMULATION RESULTS}

The proposed approach is implemented in MATLAB language on the Pentium- 4 dual core $1.66 \mathrm{GHz}$ PC. The determination of the approach control parameters is a significant problem for the CFA implementation. However, there is no formal methodology to solve the problem because different value-combinations of the parameters result in different performance of the approach. Regarding this, we have performed our parameters in such a way that we have not to update their values whenever we change the test system. In fact, preliminary numerical tests were used to set the values of the parameters and the best obtained ones are presented in Tab. 2

To investigate the efficiency and the performance of the proposed approach, a practical high-order AVR system ,as shown in Fig. 4, was tested. The parameters of the block diagram are chosen as $K_{a}=10, K_{e}=K_{g}=K_{s}=$ $1.0, \tau_{a}=0.1 \mathrm{~s}, \tau_{e}=0.4 \mathrm{~s}, \tau_{s}=0.01 \mathrm{~s}, \tau_{g}=1.0 \mathrm{~s}$. Only $K_{a}$ is load dependent, where the lower and upper bounds of the PID controller parameters are: $0 \leqslant K_{P} \leqslant 1.5$, $0 \leqslant K_{I}, K_{D} \leqslant 1.0$

In this section, we represent the terminal voltage of the AVR system with and without CFA-PID controller at first. In second, to emphasize the advantage of the proposed CFA-PID method in terms of performance, we have compared the results with the PSO-PID and the RGA-PID literature existing approach's [6] for two different value of the fitness weighting factor.

\subsection{AVR terminal voltage step response}

The terminal voltage step response of the AVR system without CFA-PID is shown in Fig. 5. In this case the system presents an undesirable oscillatory beahvior. For two fitness weighting factor, the best solutions of the AVR system with CFA-PID are summarized in Tab. 3, and the corresponding step responses are shown in Fig. 6. The curves indicate clearly that the system response is greatly improved by introducing the CFA-PID.

For the considered weighting factors, the CFA-PID parameters searching process and the convergence characteristics graphs are given in Figs. 7 and 8, respectively. As we can see from these characteristics, the approach reaches a satisfactory value without much fluctuation after 50 iterations.

\subsection{Comparison with PSO-PID controller}

For the purpose of comparison, the PSO-PID controller parameters are the same as in [6].The terminal voltage step responses of the AVR system controlled by 


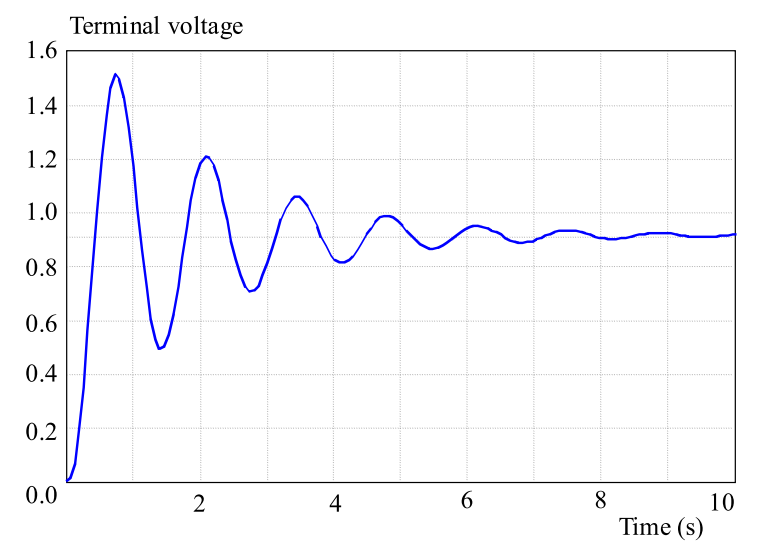

Fig. 5. Terminal Voltage step response of AVR system without PID controller

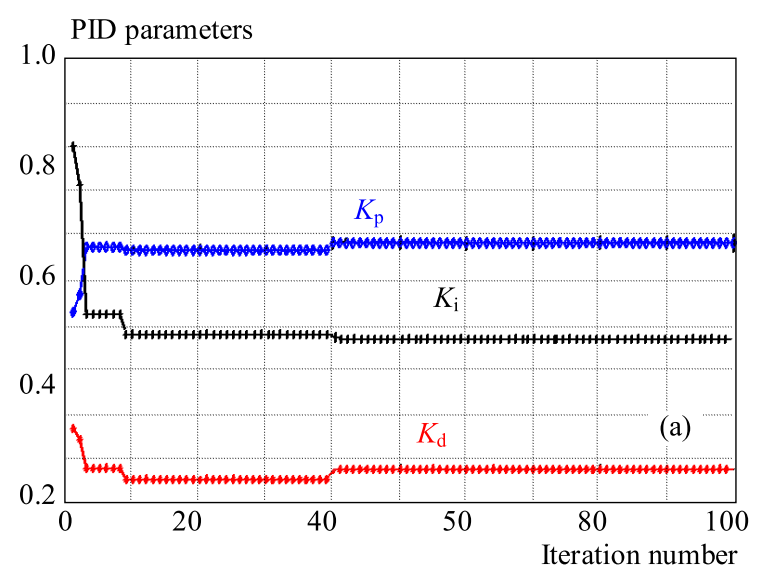

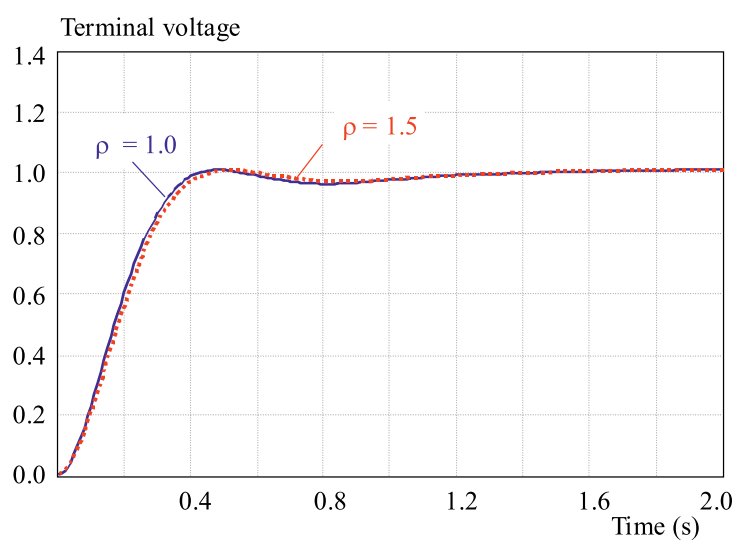

Fig. 6. Terminal Voltage step response of AVR system with CFAPID controller

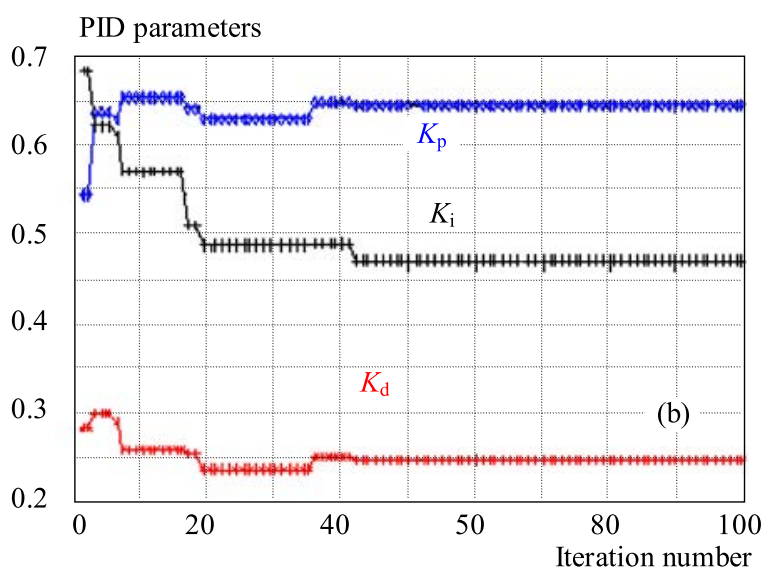

Fig. 7. CFA-PID parameters searching process over iterations: (a) $-\rho=1$, (b) $-\rho=1.5$

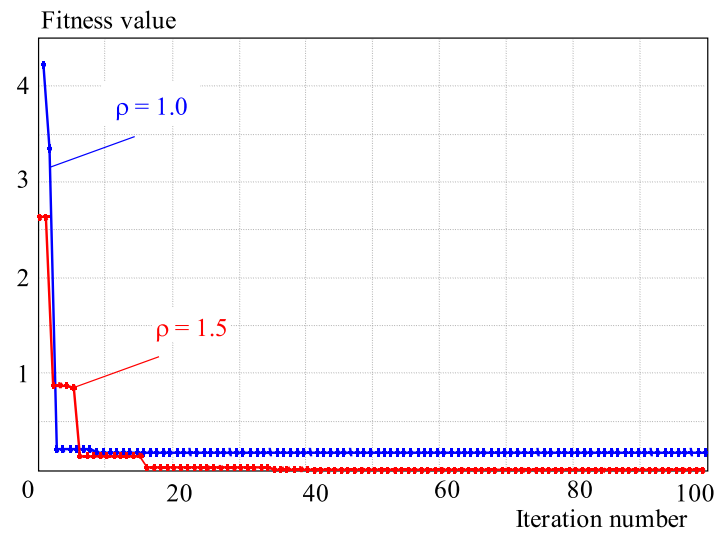

Fig. 8. Convergence characteristics of CFA-PID controller

PSO-PID controller and CFA-PID controller are shown in Fig. 9. The controller parameters and performance indices in the time domain are listed in Tab. 4.

It is observed in Fig. 9 that the CFA-PID controller has better setpoint tracking compared to PSO-PID controller. From Tab. 4, it can be stated, that the terminal voltage step response of the AVR system controlled by CFA-PID controller has smaller rising time and settling time for both weighting factor.

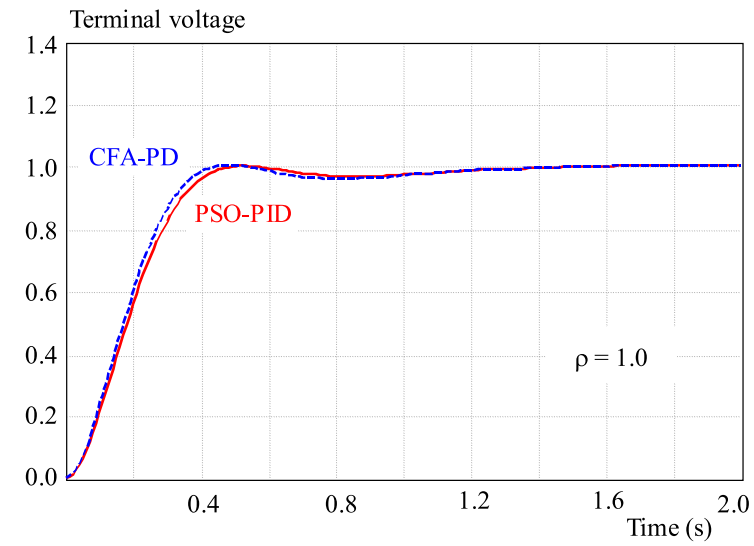

Fig. 9. Terminal Voltage step response of AVR system with CFAPID and PSO-PID controllers

\section{CONCLUSION}

This paper introduced a novel approach based on continuous firefly algorithm (CFA) for tuning the PID controller parameter in an AVR system. The obtained results through simulation experiments on a practical high order AVR system shows that the proposed method can perform an efficient search for the optimal PID controller parameters. Furthermore, the simulation results demon- 
Table 3. Best solutions using CFA-PID controller with different $\rho$

\begin{tabular}{c|ccccccccc}
\hline$\rho$ & $\begin{array}{c}\text { Number of } \\
\text { iterations }\end{array}$ & $K_{P}$ & $K_{I}$ & $K_{D}$ & $M p \%$ & $E_{s s}$ & $t_{s}$ & $t_{r}$ & $F(k)$ \\
\hline 1.0 & 100 & 0.6610 & 0.4951 & 0.2561 & 0 & 0.0051 & 0.3739 & 0.2691 & 5.8582 \\
1.5 & 100 & 0.6637 & 0.4801 & 0.2585 & 0 & 0.0025 & 0.3692 & 0.2660 & 9.2764 \\
\hline
\end{tabular}

Table 4. Best solutions using CFA-PID and PSO-PID controllers with different $\rho$

\begin{tabular}{c|ccccccccccc}
\hline$\rho$ & $\begin{array}{c}\text { Number of } \\
\text { generations }\end{array}$ & $\begin{array}{c}\text { Type of } \\
\text { controller }\end{array}$ & $K_{P}$ & $K_{I}$ & $K_{D}$ & $M p \%$ & $E_{s s}$ & $t_{s}$ & $t_{r}$ & $I T A E$ & $F(k)$ \\
\hline \multirow{2}{*}{1} & \multirow{2}{*}{100} & PSO-PID [6] & 0.6443 & 0.4700 & 0.2423 & 0 & 0 & 0.4000 & 0.2800 & 4.2040 & 5.3882 \\
& & CFA-PID & 0.6692 & 0.4959 & 0.2657 & 0 & 0.0042 & 0.3625 & 0.2610 & 4.2089 & 5.9382 \\
\hline \multirow{2}{*}{1.5} & \multirow{2}{*}{100} & PSO-PID [6] & 0.6300 & 0.4538 & 0.2276 & 0 & 0 & 0.4300 & 0.3000 & 4.1812 & 8.9322 \\
& & CFA-PID & 0.6537 & 0.4742 & 0.2467 & 0 & 0.0029 & 0.3830 & 0.2759 & 4.1627 & 9.1996 \\
\hline
\end{tabular}

strate that the new tuning method using continuous firefly algorithm can improve the control system performance in terms of time domain speicfications and setpoint tracking when compared with particle swarm optimization.

\section{REFERENCES}

[1] ELGARD, O. I. : Electric Energy Systems Theory, McGrawHill, New York, 1982, pp. 299-362.

[2] KUNDUR, P.: Power System Stability and Control, New York: McGrew Hill, USA, 1994.

[3] GAING, Z. L.: A Particle Swarm Optimization Approach for Optimum Design of PID Controller in AVR System, IEEE Transactions on Energy Conversion 19 No. 2 (2004), 348-391.

[4] KIM, D. H.-CHO, J. H.: A Biologically Inspired Intelligent PID Controller Tuning for AVR Systems, International Journal of Control Automatic and Systems 4 (2006), 624-636.

[5] MUKHERJEE, V.-GHOSHAL, S. P.: Intelligent Particle Swarm Optimized Fuzzy PID Controller for AVR System", Electric Power Systems Research 77 No. 12 (2007), 1689-1698.

[6] WONG, C. C.-LI, S. A.-WANG, H. Y.: Optimal PID Controller Design for AVR Systemjour Tamkang Journal of Science and Engineering.

[7] SHAYEGHI, H.-DADASHPOUR, J. : Anarchic Society Optimization Based PID Control of an Automatic Voltage Regulator (AVR), System, Electrical and Electronic Engineering 2 No. 4 (2012), 199-207.

[8] YANG, X. S.: Nature-Inspired Metaheuristic Algorithm, Luniver Press, 2008.

[9] YANG, X. S.: Firefly Algorithms for Multimodal Optimization, In: Watanabe, O., Zeugmann, T. (eds.) SAGA 2009 LNCS, vol. 5792, Springer, Heidelberg, 2009, pp. 169-178.
10] SAYADIA, M. K.-RAMANZIANA, R.-GHAFFARI-NASABA, N. : A Discret Firefly Meta-Heuristic with Local Search for Makespan Minimisation in Permutation Flow Shop Scheduling Problems, International Journal of Industrial Engineering Computations 1 (2010), 1-10.

[11] SEnthilnath, J.-OMKAR, S. N.-MANi, V.: Clustering using Firefly Algorithm: Performance Study, Swarm and Evolutionary Computation 1 No. 1 (2011), 164-171.

[12] GANDOMI, H.-YANG, X. S.-ALAVI, A. H.: Mixed Variable Structural Optimization using Firefly Algorithm, Computers and Structures 89 No. 23-24 (2011), 2325-2336.

[13] LUANGPAIBOON, P. : Continuous Stirred Tank Reactor Optimisation via Simulated Annealing, Firefly and Ant Colony Optimisation Elements on the Steepest Ascent, International Journal of Machine Learning and Computing 1 No. 1 (Apr 2011), 58-65.

[14] SINA, K. A.-SAIED, K. A.: Optimum Design of Structures using an Improved Firefly Algorithm", International Journal of Optimization in Civil Engineering 1 No. 2 (2011), 327-340.

Received 16 October 2012

Bendjeghaba Omar was born in 1975 in Batna, Algeria. He received his BS degree in Electromechanical Engineering from the Oils and Chemistry Institute of The University of Boumerdes (Algeria) in 1998, the MS degree from the Oils and Chemistry Institute of the University of Boumerdes (Algeria) in 2003 and the Ph.D dergree from the same university in 2010. He is currently member at the research laboratory (LREEI). His research activities include operations, control, planning and reliability of power systems, as well as evolutionary computation theory and applications. 